\title{
Segmentation of the placenta and its vascular tree in Doppler ultrasound for fetal surgery planning
}

Enric Perera-Bel, Mario Ceresa, Jordina Torrents-Barrena, Narcís Masoller, Brenda Valenzuela-Alcaraz, Eduard Gratacós, Elisenda Eixarch, Miguel A. González Ballester

\section{Enric Perera-Bel is the corresponding author. Contact: enric.perera@upf.edu}

E. Perera-Bel, M. Ceresa, J. Torrents-Barrena BCN MedTech, Department of Information and Communication Technologies, Universitat Pompeu Fabra, Barcelona, Spain.

M. A. González Ballester BCN MedTech, Department of Information and Communication Technologies, Universitat Pompeu Fabra, Barcelona, Spain. ICREA, Barcelona, Spain.

N. Masoller, B. Valenzuela-Alcaraz, Eduard Gratacós, Elisenda Eixarch BCNatal, Fetal Medicine Research Center (Hospital Clínic and Hospital Sant Joan de Déu), University of Barcelona, Barcelona, Spain; Institut d'Investigacions Biomèdiques August Pi i Sunyer (IDIBAPS), Barcelona, Spain and Centre for Biomedical Research on Rare Diseases (CIBER-ER), Madrid, Spain

\begin{abstract}
Purpose: Twin-to-Twin Transfusion Syndrome (TTTS) is a serious condition that occurs in about $10-15 \%$ of monochorionic twin pregnancies. In most instances, the blood flow is unevenly distributed throughout the placenta anastomoses leading to the death of both fetuses if no surgical procedure is performed. Fetoscopic laser coagulation is the optimal therapy to considerably improve co-twin prognosis by clogging the abnormal anastomoses. Notwithstanding progress in recent years, TTTS surgery is highly risky. Computer assisted planning of the intervention can thus improve the outcome.

Methods: In this work, we implement a GPU-accelerated random walker (RW) algorithm to detect the placenta, both umbilical cord insertions and the placental vasculature from Doppler ultrasound (US). Placenta and background seeds are manually initialized in 10-20 slices (out of 245). Vessels are automatically initialized in the same slices by means of Otsu thresholding. The RW finds the boundaries of the placenta and reconstructs the vasculature.

Results: We evaluate our semi-automatic method in 5 monochorionic and 24 singleton pregnancies. Although satisfactory performance is achieved on placenta segmentation (Dice $\geq 84.0 \%$ ), some vascular connections are still neglected due to the presence of US reverberation artifacts (Dice $\geq 56.9 \%$ ). We also compared inter-user variability and obtained Dice coefficients of $\geq 76.8 \%$ and $\geq 97.42 \%$ for placenta and vasculature, respectively. After a threeminute manual initialization, our GPU approach speeds the computation 10.6 times compared to the CPU.

Conclusions: Our semi-automatic method provides a near real-time user experience and requires short training without compromising the segmentation accuracy. A powerful approach is thus presented to rapidly plan the fetoscope insertion point ahead of TTTS surgery.
\end{abstract}

Keywords Fetal surgery · Doppler US · TTTS · placenta and vessel detection · random walker · GPU optimization 


\section{Introduction}

Fetal surgery has improved the prognosis of pregnancies with complications, and considerable progress has been evidenced in recent years. Approximately $70 \%$ of all twin pregnancies are monochorionic, i.e. the twins share the same placenta. This type of pregnancy can be risky and requires extra care and observation, as up to $25 \%$ manifest twin-to-twin transfusion syndrome (TTTS) [1,2], among other complications. In TTTS, blood is unevenly distributed between the fetuses through vascular connections (anastomoses) in the surface of the placenta. The fetuses can suffer from severe sequelae or even die if left untreated [3-5]. Fetoscopic laser coagulation is the standard treatment for TTTS, which consists on coagulating (closing) the anastomoses located on the vascular hemisphere of the placenta to restore blood flow equilibrium. Figure 1 shows an ultrasound (US) guided fetoscopic laser coagulation for TTTS treatment.

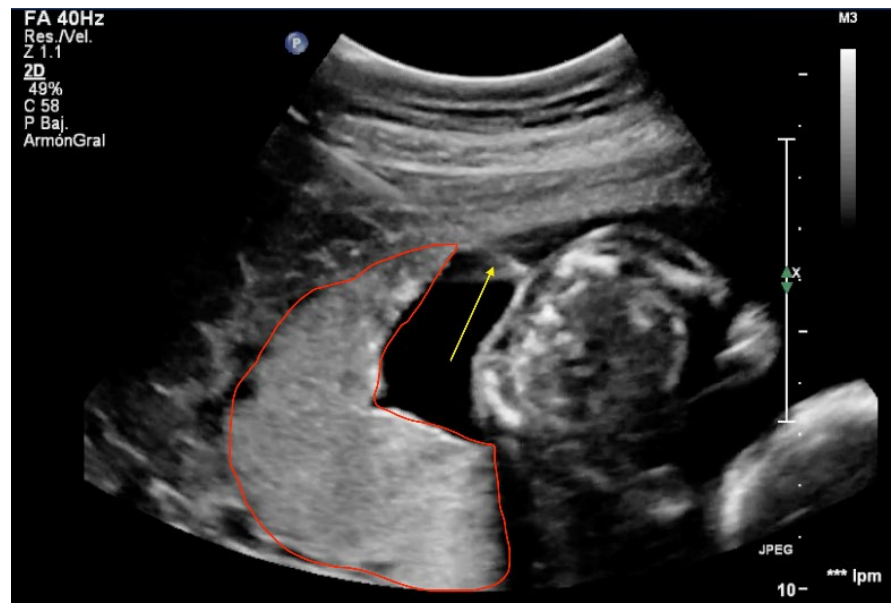

Fig. 1 US-guided fetoscopic laser coagulation. The fetoscope (yellow arrow) is inserted through the uterus to coagulate the anastomoses on the surface of the placenta (red).

In this context, three-dimensional (3D) representation of the placenta, its vasculature and the umbilical cord insertion points have the potential to significantly improve treatment outcome. Although magnetic resonance imaging (MRI) is a promising modality for revealing signs of TTTS [6], US remains the routine test in diagnosis due to its low cost and rapid acquisition. In fact, most fetal interventions are currently US guided. Additionally, Doppler US can be employed to track down and assess blood flow in the placenta and the umbilical vein and arteries. The segmentation of the aforementioned tissues from US images allows to plan the best fetoscope insertion point and quantify if all anastomoses can be reached.

Placenta detection is a difficult task due to high inter-subject variability in shape, size, position and orientation. Torrents-Barrena et al. (2019) reviewed 123 state-of-the-art segmentation and classification methodologies for the whole fetus and, more specifically, the fetal brain, lungs, liver, heart and placenta in MRI and (3D) US [6]. Because of the arbitrary position and shape that placenta takes, most of the current segmentation techniques are semi-automatic. The virtual organ computer-aided analysis (VOCAL, General Electric Healthcare, Milwaukee, WI, USA) method is based on the geometric interpolation of 2D seeded contours defined at constant intervals of a rotation angle around a predefined axis [7]. It achieved high reliability with low inter- and intra-user variability for placenta volume measurement, both ranging between 74 and $84 \%$ of agreement [8]. The random walker (RW) segmentation algorithm was also employed as a semi-automatic approach for placenta detection [9], improving accuracy over VOCAL (Dice $=86 \%)$, and in inter- and intra-subject variability ( $91 \%$ and $96 \%$ agreement, respectively). Deep learning techniques have also been explored, using recurrent neural networks and atlas based fusion methodologies to obtain Dice scores up to $86.31 \%$ of accuracy [10-12]. Nonetheless, manual annotations were also utilized to enhance these approaches. 
The latest reports on automatic MRI placenta segmentation are based on neural networks. Using a convolutional neural network (CNN) as a classifier with a conditional random field (CRF) for refinement, a Dice score of $71.95 \%$ of accuracy was obtained for healthy fetuses [13]. Alternatively, Wang et al. (2016) [14] accurately segmented the placenta (Dice $82 \%$ ) using weak annotations on a single slice, with very low inter-subject variability. The labels were propagated throughout the entire 3D stack by combining high-level features, online Random Forests and CRF. Afterwards, the same authors designed a CNN with geodesic distance transform refinement [15], with manual annotations encoded as CRF hard constraints, boosting accuracy (Dice $=89.31 \%$ ) and further reducing user influence. A fully-automatic segmentation of the placenta and its peripheral vasculature was achieved by generating a superresolution reconstruction from axial, sagittal and coronal MRI stacks, and then applying 3D Gabor filters and Support Vector Machines to segment the placenta (Dice $=82 \%$ of accuracy), and a curvature corner detector to extract the vasculature (Dice $=81 \%$ of accuracy) $[16]$.

There are mainly four categories of methodologies regarding vessel segmentation: vessel enhancement, deformable models, tracking, and machine learning [17]. Vessel enhancement rely on contrast imaging (e.g. computer tomography (CT) or MRI) to improve vessel contrast with respect to the background $[18,19]$. This approach has been used to track vasculature in mouse placenta [20,21]. Although, this approach fails to connect complex and intricate vascular trees, it is usually used as a preprocessing step to the other three categories. Deformable models can reconstruct the vasculature, given some seed points, by means of B-spline active contour a gradient field derived from the image intensity and shape constraints [22]. However, these methods are not suited for changing vessel topologies. Tracking methods also require an initialization of seeds, where a circular or elliptical cylinder is being iteratively appended in the trajectory defined by a constraint, such as energy minimization by means of graph cuts [23]. Machine learning approaches, and importantly, neural networks have been explored to successfully segment vasculature in different organs such as brain, liver and placenta from CT scans [24-27].

However, US vasculature segmentation is complex due to the low intensity contrast between tissues. Although a few studies have approached this problem for liver or kidney vasculature [28,29], there is only one precedent of US placenta vessel segmentation. Torrents-Barrena et al. (2019) [30,31] proposed the only fully automated framework for placenta and its peripheral vasculature in 3D US. The placenta was identified by means of a conditional Generative Adversial Network (Dice $=72 \%$ ), whereas the vasculature was segmented afterwards using a Modified Spatial Kernelized Fuzzy C-Means and Markov Random Fields (Dice $=68.5 \%$ ).

Our primary goal is to develop a fast, transparent, easy-to-use and robust multi-class segmentation methodology for TTTS surgery planning, which requires an accurate delineation of the placenta, its vasculature and the umbilical cord. The RW is a methodology that has successfully been used for placenta segmentation in B-mode US [9]. Here, we propose a RW-based algorithm with manual and Otsu initialization for both placenta and vasculature segmentation the first time. In contrast to the work in [9], the RW method is applied to Doppler US images. Additionally, to provide a near real-time experience to clinicians, which is key in TTTS planning, we have developed a GPU-accelerated version of the RW, with execution times similar to [30], and we have included it in a graphical user interface (GUI) adapted to clinical use. The segmentations are validated against a ground truth and inter-subject variability is also reported.

\section{Materials and Methods}

The random walker is a graph-based segmentation algorithm [32], and has derived to extended approaches [33,34]. The method segments an input image given a prior label image. It evaluates the input image as a graph, assigning a node to each pixel, and connecting the nodes by edges. According to the labelling of the prior, some nodes are labeled (seeded nodes) and some are left unlabeled (unseeded nodes). Then, it computes the probability $p_{s}$ that a random walker particle placed at an unseeded node will reach a seeded node. Since the prior contains at least two regions, each with a unique label $s$, then $p_{s}$ is computed for all labels, and the $\mathrm{s}$ with the highest probability $p_{s}$ is assigned to the evaluated unseeded node. 
This algorithm can be formulated as an electric circuit solved by superposition. For each label, the Laplace equation is solved assigning a boundary condition of $1 \mathrm{~V}$ or $0 \mathrm{~V}$ potential to the seeded nodes ( 1 for the current label, 0 for the remaining labels). A search is performed individually through all nodes to know which configuration leads to the highest potential at that node. Then, the label corresponding to that configuration is assigned to the node.

The Laplace equation can be solved implicitly as a linear system of equations.

The weight $w$ between two neighboring nodes is computed according to a Gaussian distribution:

$$
w_{i j}=e^{\left(-\beta\left(g_{i}-g_{j}\right)^{2}\right)}
$$

where $g_{i}$ and $g_{j}$ represent pixel intensities at two neighboring nodes, and $\beta$ defines the standard deviation of the distribution. Then, a node's degree $d$ is:

$$
d_{i}=\sum w_{i j}, \forall \operatorname{nodes} v_{j} \text { incident on } v_{i}
$$

$L$ is the combinatorial Laplacian matrix:

$$
L=\left\{\begin{aligned}
d_{i}, & \text { if } i=j \\
-w_{i j}, & \text { if } v_{i} \text { and } v_{j} \text { are neighbors } \\
0, & \text { otherwise }
\end{aligned}\right.
$$

By reordering $L$, we obtain a square matrix for the marked (labeled) nodes $L_{M}$ and a square matrix for the unlabeled nodes $L_{U}$. $B T$ is the transpose of $B$, which are the remaining elements of $L$ after reordering:

$$
L=\left[\begin{array}{cc}
L_{M} & B \\
B^{T} & L_{U}
\end{array}\right]
$$

$M$ is a matrix that specifies for which label $s$ the system is solved by:

$$
M_{i s}=\left\{\begin{array}{l}
1, \text { if } v_{i} \text { has label } s \\
0, \text { if } v_{i} \text { does not have label } s
\end{array}\right.
$$

Finally, the linear system is

$$
L_{U} x_{U}=-B^{T} M
$$

where $x_{U}$ are the probabilities of the unmarked nodes, which has $N$ rows (number of unlabeled nodes) and $S$ columns (number of labels). Since every row of $x_{U}$ adds to unity, the last column can be obtained by subtraction, thus solving $S-1$ linear systems.

\subsection{Implementation}

The only user-dependent parameter of the RW is $\beta$, which is employed during the edge weight computation and defined by a Gaussian distribution (see Equation 1). $\beta$ is also defined as the inverse of twice the variance:

$$
\beta=\frac{1}{2 \sigma^{2}}
$$

Lower $\sigma$ enhances intensity similarities over the image, whereas higher $\sigma$ increases the geometrical relevance of the prior labelling. Visual inspection of the images reveals that weak boundaries have an intensity gradient of 5 gray levels. Thus, $3 \sigma$ is set to $5(\beta=0.18)$ to reduce user-dependency while maintaining a high boundary adherence. 
The algorithm has been implemented in $\mathrm{C}++$ using ITK ${ }^{1}$ [35] and Eigen $3^{2}$ [36]. To speed up the computation, the image size, and consequently, the linear system, is cropped by adjusting the region of interest to the placenta area. We use the biconjugate gradient stabilized method (BiCGStab) [37] along with Jacobi preconditioning to find an optimal solution for $L_{U}$. Since the linear system can be defined with 10-100 million unknowns, a GPUaccelerated RW has been approached. The GPU BiCGStab solver was implemented using CUDA3 (CUBLAS and CUSPARSE). Both CPU and GPU RW workflows are displayed in Table 1. The CPU and GPU used are, respectively, an Intel i7-7700 CPU and a GTX $1080 \mathrm{Ti}$. To facilitate the portability of our RW implementation, we have also developed a plugin for the Medical Imaging Interaction Toolkit (MITK) ${ }^{4}$ with a clear and concise graphical user interface (Figure 2). A stable version is available at: github.com/enricperera/mitkRWSegmentationPlugin.

Table 1 Workflow of the RW algorithm for CPU and GPU implementations

\begin{tabular}{l|c}
\multicolumn{1}{c}{ CPU } & GPU \\
\hline $\begin{array}{l}\text { 1. Crop image according to } \\
\text { prior labelling }\end{array}$ & \\
2. Identify labeled and & \\
unlabeled nodes & \\
3. Build linear system & \\
$\left(L_{U}\right.$ and $\left.B^{T}\right)$ & \\
4. Solve linear system & 4. Solve linear system \\
4.1 CPU BiCGStab & 4.1 Copy linear system \\
& to device \\
& 4.2 GPU BiCStab \\
& 4.3 Copy linear system \\
& solution to host \\
5. Build segmentation from & \\
prior and linear system & \\
solution &
\end{tabular}

${ }^{1}$ The Insight Segmentation \& Registration Toolkit (ITK): https://itk.org

2 Eigen3: http://eigen.tuxfamily.org

${ }^{3}$ CUDA: https://developer.nvidia.com/cuda-toolkit

${ }^{4}$ The Medical Imaging Interaction Toolkit (MITK): http://mitk.org 


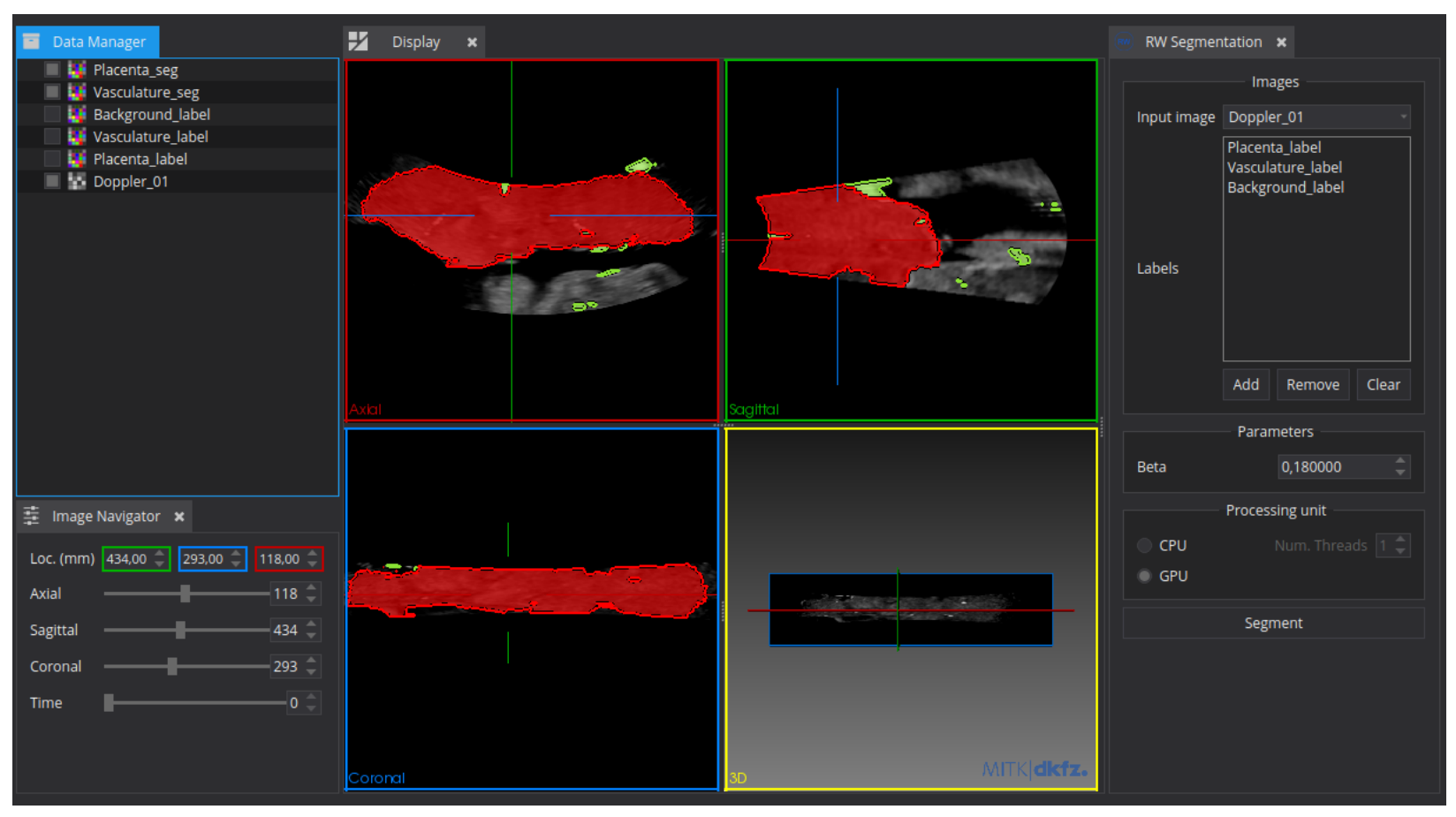

Fig. 2 Screenshot of the RW GUI. The proposed plugin is displayed in the right column. An input image and the labels must be specified, as well as the beta parameter and the computation engine (CPU/GPU).

\subsection{Placenta and vessel segmentation}

The proposed methodology consists of two RW iterations which are performed to reduce user interaction without compromising accuracy. After an initial manual labelling, the whole process is executed automatically. The first iteration is used to obtain a rough approximation of the placenta volume whereas the second iteration combines the aforementioned rough segmentation with the initial detection of the vasculature. The workflow is depicted in Algorithm 1 and Fig. 3.

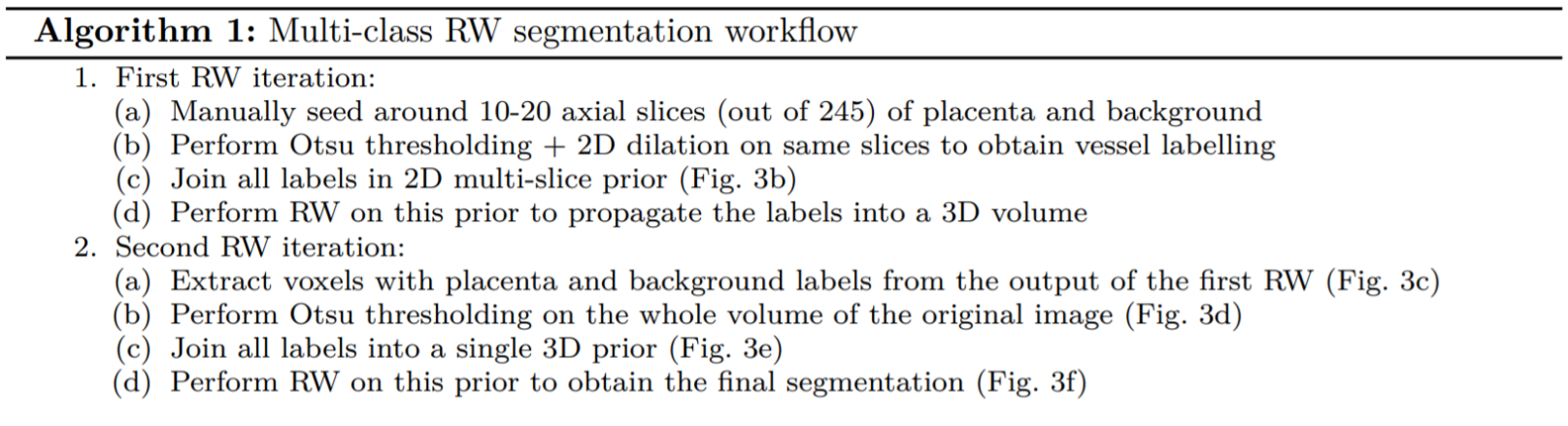




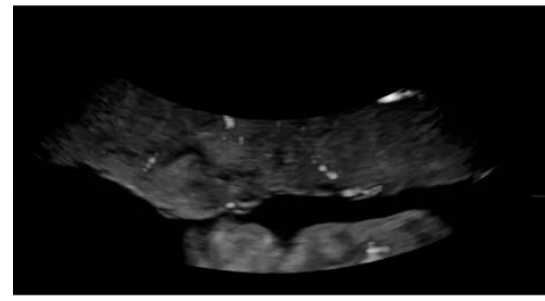

(a)

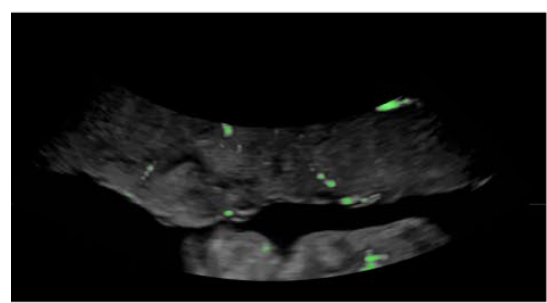

(d)

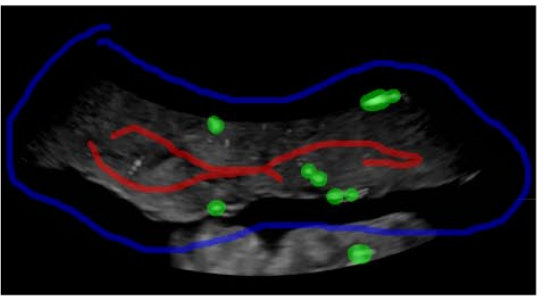

(b)

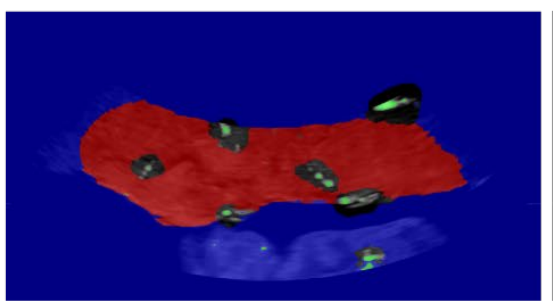

(e)

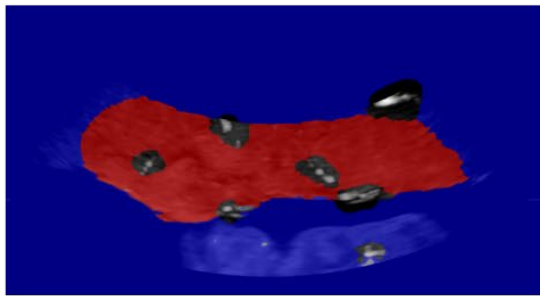

(c)

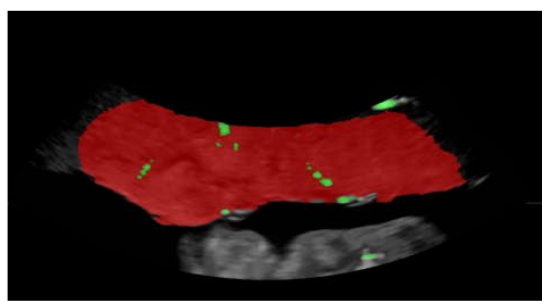

(f)

Fig. 3 Prior initialization and fusion workflow. Placenta, vessels and background are represented in red, green and blue, respectively. a) Doppler US image, b) 1st iteration prior, c) placenta and vessel mask of the 1st RW output, d) vasculature Otsu labelling, e) 2nd iteration prior, and f) segmentation of the placenta and its vasculature.

The prior for the first iteration consists on labelling a set of axial slices through the entire stack. A labelling protocol similar to the one proposed by Stevenson et al. (2015) [9] is established. The slice previous to the initial slice with placental zones is seeded as background. The next tenth slice (towards the center of the placenta) is also seeded. The same procedure is performed at the other extreme of the volume. The remaining volume is labeled every twentieth slice. On each of these slices, two different regions are manually labeled: placenta and background. Vessels are then labelled automatically on the same slices as placenta by means of Otsu thresholding. A 2D morphological dilation is finally applied to vessels to avoid the propagation of the estimated placenta mask towards other image regions.

The second round starts with the rough placenta mask computed on the first iteration, which is subsequently combined with a volumetric prior of the vessels. This time, Otsu thresholding is applied through the entire stack to obtain the vasculature labelling. Pixels with overlapping labels are left unseeded. The RW is thus computed again with the new prior to obtain the final placenta and vessel segmentation.

\section{Results}

\subsection{Segmentation accuracy}

The data collected is composed of 5 monochorionic and 24 singleton pregnancies with 3D Doppler HD-flow US acquisitions obtained from BCNatal. The equipment used was a Voluson E10 from GE Healthcare, with probe eM6C (curved electronic matrix 4D). The gestational age of pregnant women range from 16 to 36 weeks. The images were converted to gray scale for analysis.

Visual inspection reveals that our placenta segmentation is accurate (see Fig. 4). The segmentation of this organ with the amniotic fluid is well-defined, but some borders with low texture gradient are miss-segmented (see Fig. 4 second and fourth rows). Additionally, some US stacks present shadows, but the RW performs successfully if texture information is not fully hidden (see Fig. 4 second row). The boundaries of hyperintense vessels are precisely delineated, but the RW fails to reconstruct the whole vascular tree. Moreover, fetus vasculature is also segmented as well as noise derived from low speed amniotic fluid motion due to its resemblance with hyperintense vessels (false positives). 
In $75 \%$ of singleton cases, the umbilical cord insertion and the thick vessels that emanate from it are accurately detected (see Fig. 4 first to forth rows). In monochorionic pregnancies, both umbilical cord insertions are detected in two out of five cases (see Fig. 4 fifth row). Only one cord insertion was localized on the remaining three cases.
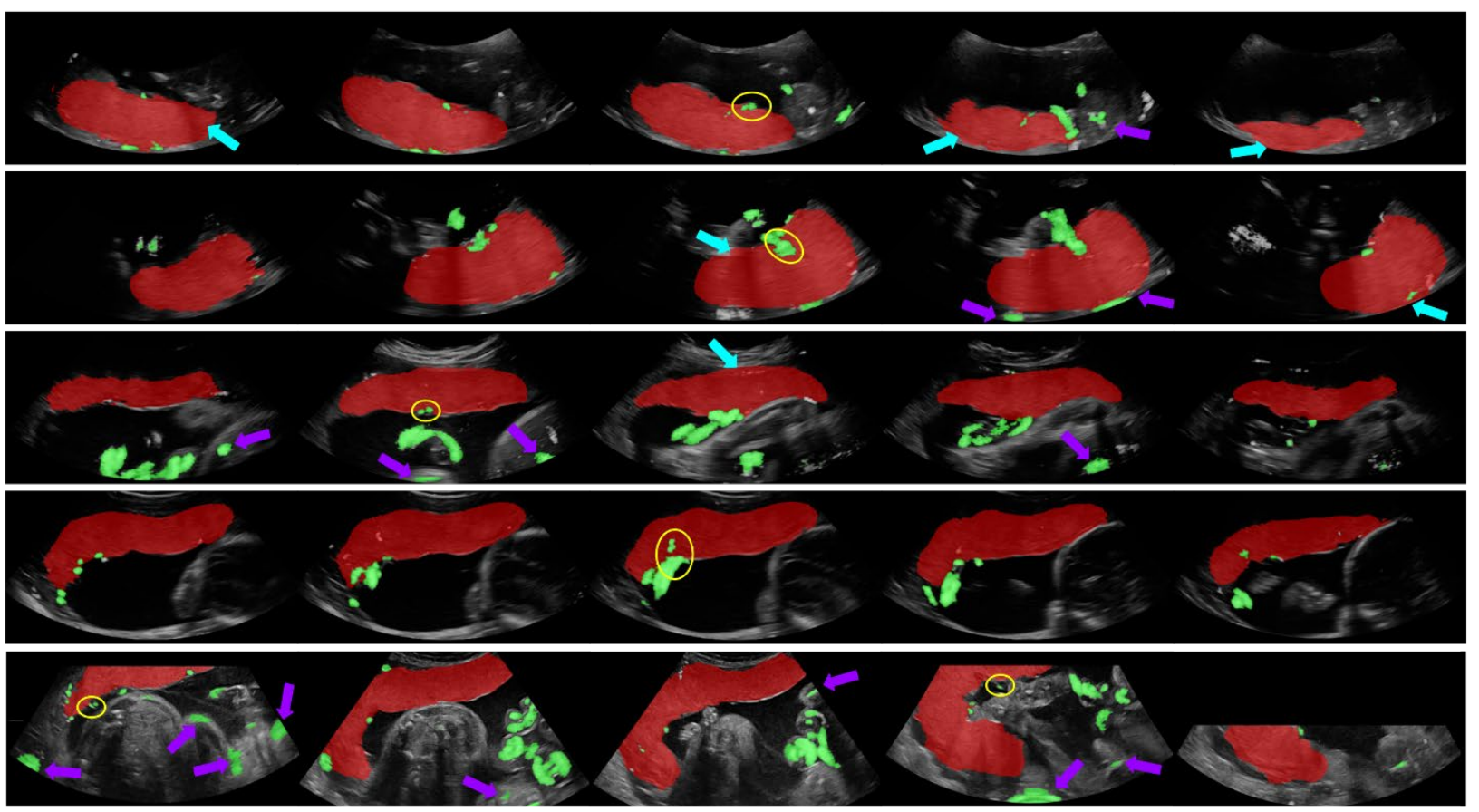

Fig. 4 Multi-class segmentation of five cases (rows) with five different slices (columns). Placenta and its vasculature are shown in red and green, respectively. Cyan arrows show false positives of the placenta. Purple arrows indicate false positives of the vasculature. Yellow circles indicate the umbilical cords insertions.

Three different users, two clinicians (NM and BVA) and one non-clinician (EPB), independently labelled the RW prior for all the 29 datasets. Additionally, manual annotation by EPB was taken as ground truth for 20 axial slices per dataset (out of the 245 each image has) randomly separated from 1 to 30 slices and ensuring placenta was visible in all of them. The annotations were revised to fine-tune the borders with features identified by the clinicians NM and BVA. Dice and Jaccard coefficients, and volumetric similarity (VS) were computed against the ground truth, which are defined

$$
\begin{aligned}
& \text { Dice }=\frac{2\left|V_{S} \cap V_{G T}\right|}{\left|V_{S}+V_{G T}\right|} \\
& \text { Jaccard }=\frac{\left|V_{S} \cap V_{G T}\right|}{\left|V_{S} \cup V_{G T}\right|} \\
& V S=1-\frac{\left|V_{S}-V_{G T}\right|}{\left|V_{S}+V_{G T}\right|}
\end{aligned}
$$

with $V_{S}$ and $V_{G T}$ being the segmentation volume and the ground truth volume, respectively. In singleton patients, the segmented placenta scored an average Dice, Jaccard and VS of $85.5 \%, 74.9 \%$ and $93.5 \%$, respectively, and $84.0 \%$, $72.7 \%$, and $91.6 \%$ in monochorionic cases. Vessel reconstruction registered, in the same order, $58.4 \%, 43.9 \%$ and $69.9 \%$ in singletons, and $56.9 \%, 40.4 \%$, and $66.2 \%$ in twins. The individual coefficients for each user are represented in Table 2. Additionally, due to the lower accuracy of the vessel segmentation, we performed manual corrections in the 5 monochorionic cases and obtained a significant improvement (Dice $=86.4 \pm 3.7 \%$, Jaccard $=76.0 \pm 5.7 \%$ and $\mathrm{VS}=95.1 \pm 3.2 \%$ ). 
Table 2 Segmentation accuracy of singleton and monochorionic pregnancies (average \pm standard deviation)

\begin{tabular}{cccc|ccc} 
& \multicolumn{3}{c}{ Singleton pregnancies } & \multicolumn{3}{c}{ Monochorionic pregnancies } \\
Placenta & Dice & Jaccard & VS & Dice & Jaccard & VS \\
\hline NM & $88.0 \pm 3.7$ & $78.7 \pm 5.7$ & $96.1 \pm 3.8$ & $83.0 \pm 3.9$ & $71.2 \pm 5.7$ & $89.2 \pm 5.3$ \\
BVA & $85.4 \pm 4.2$ & $74.7 \pm 6.5$ & $92.1 \pm 4.8$ & $85.8 \pm 3.5$ & $75.3 \pm 5.5$ & $95.1 \pm 2.8$ \\
EPB & $83.0 \pm 5.4$ & $71.3 \pm 7.6$ & $92.5 \pm 5.4$ & $83.1 \pm 4.6$ & $71.4 \pm 6.8$ & $90.6 \pm 5.9$ \\
Vasculature & & & & & & \\
\hline NM & $59.0 \pm 20.6$ & $44.8 \pm 20.4$ & $70.5 \pm 22.6$ & $57.6 \pm 10.5$ & $41.2 \pm 9.8$ & $66.0 \pm 10.9$ \\
BVA & $58.4 \pm 19.9$ & $44.0 \pm 19.4$ & $70.4 \pm 22.3$ & $56.8 \pm 10.9$ & $40.4 \pm 9.8$ & $66.0 \pm 10.7$ \\
EPB & $57.6 \pm 18.7$ & $42.9 \pm 18.4$ & $68.8 \pm 21.4$ & $56.2 \pm 10.3$ & $39.8 \pm 9.4$ & $66.6 \pm 11.6$
\end{tabular}

Additionally, Dice and Jaccard coefficients, and VS were computed between the segmentations from the three users. The reported segmentation results show relatively low variability rates between expert and amateur users, both in singleton and monochorionic cases (see Table 3). Placenta detection presents good agreement between clinicians (Dice $\geq 78.7 \%$ ), and the non-clinician user (Dice $\geq 76.8 \%$ ). In the same line, vessel segmentation performance is similar among users (Dice $\geq 96.5 \%$ ).

Table 3 Inter-user segmentation variability of singleton and monochorionic pregnancies (average \pm standard deviation)

\begin{tabular}{cccc|ccc} 
& \multicolumn{3}{c}{ Singleton pregnancies } & \multicolumn{3}{c}{ Monochorionic pregnancies } \\
Placenta & Dice & Jaccard & VS & Dice & Jaccard & VS \\
\hline NM-EPB & $80.4 \pm 6.5$ & $67.7 \pm 9.0$ & $92.0 \pm 7.9$ & $83.4 \pm 2.0$ & $71.5 \pm 2.9$ & $95.2 \pm 4.6$ \\
BVA-EPB & $76.8 \pm 7.2$ & $62.9 \pm 9.8$ & $89.0 \pm 9.2$ & $82.0 \pm 2.2$ & $69.9 \pm 3.1$ & $90.0 \pm 6.0$ \\
BVA-NM & $84.4 \pm 5.7$ & $73.4 \pm 8.5$ & $91.4 \pm 7.0$ & $78.7 \pm 2.2$ & $64.9 \pm 2.9$ & $85.7 \pm 2.3$ \\
Vasculature & & & & & & \\
\hline NM-EPB & $97.4 \pm 1.4$ & $86.1 \pm 2.2$ & $98.7 \pm 1.4$ & $97.3 \pm 1.5$ & $88.5 \pm 2.4$ & $99.0 \pm 0.8$ \\
BVA-EPB & $97.6 \pm 1.3$ & $86.1 \pm 2.0$ & $99.1 \pm 1.1$ & $96.7 \pm 1.4$ & $85.1 \pm 2.1$ & $97.6 \pm 0.6$ \\
BVA-NM & $98.8 \pm 0.6$ & $91.3 \pm 1.0$ & $99.4 \pm 0.6$ & $96.5 \pm 1.8$ & $85.7 \pm 2.8$ & $97.2 \pm 1.8$
\end{tabular}

\subsection{Segmentation time}

The workflow of this multi-class segmentation process is divided mainly into two steps, manual prior initialization and algorithm computation. The former takes an average of $271 \pm 71 \mathrm{~s}$ for EPB, $198 \pm 53 \mathrm{~s}$ for NM, and $209 \pm 37 \mathrm{~s}$ for BVA, whereas the computation time takes $444 \pm 146,528 \pm 136 \mathrm{~s}$ and $502 \pm 173 \mathrm{~s}$, respectively. There is no labelling time difference between singleton and twin cases. The entire workflow period of the methodology takes nearly around 715,726 and 710 seconds for EPB, NM and BVA, respectively. The manual correction of the vasculature segmentation was only performed by EPB and it took $32.2 \pm 7.9 \mathrm{~min}$. 
Table 4 shows that our GPU implementation speeds up the computation 10.6 times compared to CPU. Although a higher performance ratio (x11.8) is obtained in the second iteration, the first round leads to an overall reduction of more than 400 seconds. Extra time is consumed during the memory allocation, and data copy to the graphics card, which takes $3.74 \pm 0.99$ and $1.37 \pm 0.06$ seconds for the first and second iteration, respectively. Table 3 shows the total usage in terms of host (CPU) and device (GPU) memory. The largest amount of memory consumption depends on the first iteration. The CPU implementation uses a maximum host memory of $8.7 \pm 2.4 \mathrm{~GB}$, whereas the GPU utilizes $8.5 \pm 2.3 \mathrm{~GB}$ and $6.6 \pm 2.3 \mathrm{~GB}$ of host and device memory, respectively. The device only stores the data related to the linear system, which is the most memory intensive task (around $75 \%$ ).

Table 4 Computation time (s) and memory usage (GB) of BiCGStab solver for CPU and GPU for the first and second iterations of the RW segmentation workflow (Algorithm 1), and for the overall process (average \pm standard deviation)

\begin{tabular}{lll|ll} 
& & System & \multicolumn{2}{c}{ Memory } \\
& & solving (s) & Host & Device \\
\hline \multirow{2}{*}{\begin{tabular}{ll|ll} 
1st \\
iter.
\end{tabular}} & CPU & $449.01 \pm 145.58$ & $8.7 \pm 2.4$ & GPU \\
& speed up & $42.65 \pm 15.41$ & $8.5 \pm 2.3$ & $6.6 \pm 2.3$ \\
\hline \multirow{2}{*}{ 2nd } & CPU & $30.14 \pm 30.23$ & $7.1 \pm 1.9$ & $\mid$ \\
iter. & GPU & $2.55 \pm 2.87$ & $6.8 \pm 1.7$ & $5.3 \pm 1.9$ \\
& speed up & 11.8 & & \\
\hline & CPU & $479.15 \pm 175.81$ & $8.7 \pm 2.4$ & $\mid$ \\
Total & GPU & $45.20 \pm 18.28$ & $8.5 \pm 2.3$ & $6.6 \pm 2.3$ \\
& speed up & 10.6 & &
\end{tabular}

\section{Discussion \\ 4.1. Segmentation accuracy}

The reported results were computed on a ground truth of 20 randomly selected axial slices per placental volume (i.e. a total of 580 annotated slices). Considering that the proposed methodology is semiautomatic, with annotations on 10 to 20 slices, the algorithm should perform consistently and obtain an accurate segmentation on the global scale (as reported in a similar imaging modality in [9]). Thus, and given that our dataset is very large, we consider that the validation methodology proposed is sufficient for the current scenario. However, this validation procedure has a clear limitation as local features are not being evaluated.

On one hand, the comparison of the proposed methodology with a ground truth reveals a high accuracy on placenta segmentation for both singleton (Dice $=85.5 \%$ ), and monochorionic (Dice $=84.0 \%$ ) pregnancies. The segmentation is clearly delineated against most of the surrounding tissues. However, the contact between fetus and placenta, or uterus and placenta, can be mis-segmented because the texture of both tissues is very similar (Figure 4 cyan arrows). Nevertheless, even expert clinicians state the difficulty of precisely locating these boundaries in US. These results are in concordance with the literature, where semi-automatic approaches outperform automatic ones: Stevenson et al. (2015) [9] report a Dice coefficient of $87 \%$ in B-mode US singleton images using also a RW approach, and Torrents et al. (2019) [30] reported the use of a fully automatic Neural Network approach, also on B-mode US, with a $72 \%$ Dice score.

On the other hand, vasculature segmentation with the proposed methodology leads to lower values (Dice $=58.4 \%$ in singletons and Dice $=56.9 \%$ in monochorionic twins). Although for thin structures, such as vessels, Dice metrics are known to report lower values, as small perturbations in the segmentation lead to large variations, there are some 
limitations in the proposed methodology that are reflected with the reported Dice scores. The first one is that all vessels are detected by Otsu thresholding, not only the umbilical cord and the placenta vasculature, but also fetus's veins and arteries. The second one is due to a Doppler ultrasonography limitation; Doppler US allows the visualization of the blood flow as hyperintense data unless the flow is parallel to the probe, in which case the region remains hypointense. Otsu thresholding is a great approach to detect the vasculature in this type of imaging, but neither Otsu nor RW can track disconnected hypointense vessels. Additionally, Doppler US in the uterus is highly susceptible to amniotic fluid waves; hyperintense waves that cover a big portion of the image and that are wrongly initialized as vessel (worst performing case gets a $31.7 \%$ Dice score). Thus, Otsu thresholding proves not to be an optimal initialization method for noisy images and/or with disconnected vascular tree. Nonetheless, for clear images the proposed methodology is a valid approach, as we also achieved Dice coefficients up to $87.1 \%$ in vasculature segmentation.

A low variability among users for both placenta (Dice $\geq 78.7 \%$ ) and vessels (Dice $\geq 96.5 \%$ ) segmentation was achieved. Placenta segmentation performs consistently, and minimal effect is found in the vasculature due to an automatic, Otsu, initialization. These results prove that RW is an effective methodology for placenta segmentation, not only in B-mode US, as reported by Stevenson et al. (2015) [9] with a 91\% Dice agreement, but also in Doppler ultrasonography. Additionally, fine-tuning $\beta$ parameter to guarantee boundary adherence across different imaging sites should be further explored.

Clinicians observed that in monochorionic pregnancies $70 \%$ of the umbilical cord insertions are detected (both cords in two cases, one cord in the other three), and $75 \%$ in singletons. The cords that are not detected are an imaging drawback, rather than an algorithmic one. Cords can be hidden between the fetus and the placenta or can fall outside the US field-of-view. This anatomical landmark could be used to locate the vascular hemisphere. Hence, the incision point of the fetoscope can be planned to rapidly burn the abnormal connections, which will significantly reduce the intervention time and risks, even if the exact anastomoses to coagulate are not specifically detected.

\subsection{Segmentation time}

Cheong et al. (2010) [8] performed placenta measurements using VOCAL. Authors needed 6.2 minutes on average to delineate the contours of 10 US slices. Considering that we label from 10 to 15 US slices in 3.2-4.5 minutes, an improvement is clearly made over VOCAL on the manual initialization procedure. These results are is also in line with the $2.9 \pm 0.5$ minutes needed for RW initialization in [9]. However, we report a much higher computation time of $449 \mathrm{~s}$, compared to $43.6 \mathrm{~s}$, which could be explained by the large size of our dataset ( $948 \times 718 \times 245$ pixels). Note that the time to find an optimal solution is directly proportional to the image size.

Gokławski et al. (2015) [38] proposed a RW GPU implementation. However, they reduced the graph size through super-pixel techniques due to GPU memory restrictions. Therefore, the RW capability to correctly evaluate weak boundaries was somewhat lost. For datasets with 28-88 million voxels, authors reported RW computation times of 1447 seconds. Although our approach requires around $8.5 \mathrm{~GB}$ and $6.6 \mathrm{~GB}$ of host and device memory, it provides similar times for similar sized datasets (11-69 seconds for stacks with 28-103 million voxels) without data reduction. Additionally, the fully automatic approach of Torrents et al. (2019) [30] takes 45 seconds for placenta and 5 minutes for the vasculature, which is similar to ours that also takes around 5 minutes for the manual initialization and the computation.

\subsection{TTTS treatment planning}

TTTS is a critical condition for the fetuses that needs to be treated as soon as possible after diagnosis, ideally within first $24 \mathrm{~h}$ after diagnosis in more severe cases. Once the patient has given consent for surgery, it takes around 1 hour to prepare the operation room. Thus, there is a short period of time to perform treatment planning, hence (near) realtime performance is needed. On one hand, manual segmentation is too cumbersome. On the other hand, our methodology (manual initialization + computation) takes around 12 minutes using a CPU as a computation engine, and only 5 minutes with the GPU. However, it leaves some placental surface vessels unconnected and it is quite 
susceptible to noise (see section 4.1), so manual corrections must be performed. Whereas 4 out of 5 cases had an initial accuracy of Dice $\geq 59.6 \%$ and took 30 minutes or less to revise, the other one took 43.6 minutes (Dice $=38.2 \%$ ). For the first scenario the total segmentation time (manual initialization, algorithm execution, and manual corrections) is around 40-45 minutes, which leaves just enough time to plan the intervention (15-20 minutes). However, we observe that in the second scenario, with low initial accuracies (Dice $<50 \%$ ), there is not enough time left to plan the intervention. Thus, it would be preferable to skip the corrections and consider only the detected umbilical cord's insertion points, as the fetoscope insertion can still be planned even if the exact anastomoses are not specifically detected pre-operatively.

The work of Stevenson et al. (2015) [9] proposed an accurate methodology to segment the placenta. However, their goal was not focused on TTTS and, thus, vasculature segmentation was not explored. We have extended their approach enabling it for fetoscopic laser coagulation planning, with vasculature segmentation and umbilical cord insertion point detection. An already existing tool for TTTS planning, with a promising fully automatic workflow, is that of Torrents et al. (2019) [30]. However, it requires of MRI and (optionally) US and US Doppler to enhance vasculature segmentation. Considering that US is the standard procedure for TTTS diagnosis and that MRI is not required, our simpler approach makes it possible to plan the intervention in those centers that do not include MRI in their standard protocol for fetal pathologies.

\section{Conclusions}

We have proposed a GPU-accelerated scheme for multi-class US segmentation to speed up the TTTS surgical planning. Our RW implementation is generic in the sense that it can be used among doctors with different levels of expertise to segment the placenta, its vasculature and both umbilical cord insertions. Even though we did not specifically extract the abnormal anastomoses but the whole vascular tree, a placental map is rendered which can be extremely useful for TTTS treatment planning. A low inter-user variability, a fast initialization and an optimized GPU computation allow for a rapid decision of fetoscope insertion point and to plan the path to reach all the anastomoses. The proposed RW methodology can be potentially used for treatment planning of other fetal diseases and pathologies.

Future work should cover the following limitations. Firstly, a noise filtering scheme is required to reduce the sensitivity of the Doppler effect and improve vasculature detection. Since US images are corrupted with speckle and reverberation noise inherently, blood vessels are occasionally confused with this sort of artifacts. Secondly, the peripheral anastomoses need to be selected from the entire vasculature to efficiently plan the TTTS surgery. Thirdly, although the manual initialization time (around 4 minutes) is acceptable for use in daily clinical practice, a faster and/or automatic approach should be considered.

\section{Acknowledgements}

The research leading to these results has received funding by The Cellex Foundation, "LaCaixa" Foundation under grant agreements LCF/PR/GN14/10270005 and LCF/PR/GN18/10310003, AGAUR under grant 2017 SGR n ${ }^{\circ} 1531$, the Spanish Ministry of Economy and Competitiveness under the María de Maeztu Units of Excellence Programme [MDM-2015-0502], and the European Commission under the H2020 ATTRACT project MIIFI. Additionally, Enric Perera-Bel has received funding from the Spanish Ministry of Economy and Competitiveness under the Programme for the Formation of Doctors [BES-2017-081164], and Elisenda Eixarch from the Departament de Salut under grant SLT008/18/00156.

\section{Conflict of interest}

The authors declare that they have no conflict of interest.

\section{Ethical approval}


All procedures performed in studies involving human participants were in accordance with the ethical standards of the institutional and/or national research committee and with the 1964 Helsinki Declaration and its later amendments or comparable ethical standards (PIC-39-16, HCB/2016/0138).

\section{Informed consent}

Informed consent was obtained from all individual participants included in the study

\section{References}

1. Benirschke K (1995) The biology of the twinning process: How placentation influences outcome. Semin. in Perinatol. 19:342-350

2. Denbow ML, Cox P, Taylor M, Hammal DM, Fisk NM (2000) Placental angioarchitecture in monochorionic twin pregnancies: Relationship to fetal growth, fetofetal transfusion syndrome, and pregnancy outcome. Am. J. of Obstet. and Gynecol. 182:417-426

3. Haverkamp F, Lex C, Hanisch C, Fahnenstich H, Zerres K (2001) Neurodevelopmental risks in twin-to-twin transfusion syndrome: preliminary findings. Eur. J. of Paediatr. Neourol. 5:21-27

4. Rossi AC, D'Addario V (2008) Laser therapy and serial amnioreduction as treatment for twin-twin transfusion syndrome: a metaanalysis and review of literature. Am. J. of Obstet. and Gynecol. 198:147-152

5. Senat MV, Deprest Jan, Boulvain M, Paupe A, Winer N, Ville Y (2004) Endoscopic Laser Surgery versus Serial Amnioreduction for Severe Twin-to-Twin Transfusion Syndrome. N. Engl. J. of Med. 351:136-144

6. Torrents-Barrena J, Piella G, Masoller N, Gratacós E, Eixarch E, Ceresa M, González Ballester MA (2019) Segmentation and classification in MRI and US fetal imaging: Recent trends and future prospects. Med. Image Anal. 51:61-88

7. Collins SL, Stevenson GN, Noble JA, Impey L (2013) Rapid Calculation of Standardized Placental Volume at 11 to 13 Weeks and the Prediction of Small for Gestational Age Babies. Ultrasound in Med. \& Biology $36: 253-260$

8. Cheong KB, Leung KY, Li TKT, Chan HY, Lee YP, Tang MHY (2010) Comparison of inter- and intraobserver agreement and reliability between three different types of placental volume measurement technique (XI VOCAL ${ }^{\mathrm{TM}}$, VOCAL ${ }^{\mathrm{TM}}$ and multiplanar) and validity in the in-vitro setting. Ultrasound in Obstet. and Gynecol. 36:210-217

9. Stevenson GN, Collins SL, Ding J, Impey L, Noble JA (2015) 3-D Ultrasound Segmentation of the Placenta Using the Random Walker Algorithm: Reliability and Agreement. Ultrasound in Med. \& Biology 41:31823193

10. Looney P, Stevenson GN, Nicolaides KH, Plasencia W, Molloholli M, Natsis S, Collins SL (2017) Automatic 3D ultrasound segmentation of the first trimester placenta using deep learning. 2017 ISBI 2017 279-282

11. Yang X, Yu L, Li S, Wen H, Luo D, Bian C, Qin J, Ni D, Heng PA (2019) Towards Automated Semantic Segmentation in Prenatal Volumetric Ultrasound. Trans. on Med. Imaging 38:180-193

12. Oguz BU, Wang J, Yushkevich N, Pouch A, Gee J, Yushkevich PA, Schwartz N, Oguz I (2018) Combining Deep Learning and Multi-atlas Label Fusion for Automated Placenta Segmentation from 3DUS. In: Int. Workshop on Preterm, Perinatal and Paediatr. Image Anal., Springer, Cham, pp 138-148

13. Alansary A, Kamnitsas K, Davidson A, Khlebnikov R, Rajchl M, Malamateniou C, Rutherford M, Hajnal JV, Glocker B, Rueckert D, Kainz B (2016) Fast Fully Automatic Segmentation of the Human Placenta from Motion Corrupted MRI. Int. Conference on Med. Image Comput. and Comput. Assist. Interv. 589-597.

14. Wang G, Zuluaga MA, Pratt R, Aertsen M, Doel T, Klusmann M, David AL, Deprest J, Vercauteren T, Ourselin S (2016) Slic-Seg: A minimally interactive segmentation of the placenta from sparse and motioncorrupted fetal MRI in multiple views. Med. Image Anal. 34:137-147

15. Wang G, Zuluaga MA, Li W, Pratt R, Patel PA, Aertsen M, Doel T, David AL, Deprest J, Ourselin S, Vercauteren T (2018) DeepIGeoS: A Deep Interactive Geodesic Framework for Med. Image Segmentation. Trans. on Pattern Anal. and Mach. Intell. 41:1559-1572 
16. Torrents-Barrena J, Piella G, Masoller N, Gratacós E, Eixarch E, Ceresa M, González Ballester MA (2019) Fully automatic 3D reconstruction of the placenta and its peripheral vasculature in intrauterine fetal MRI. Med. Image Anal. 54:263-279

17. Moccia S, De Momi E, El Hadji S, Mattos LS (2018) Blood vessel segmentation algorithms - Review of methods, datasets and evaluation metrics. Comput. Methods and Programs in Biomed. 158:71-91

18. Selle D, Preim B, Schenk A, Peitgen HO (2002) Analysis of vasculature for liver surgical planning. Trans. on Med. Imaging 21:1344-1357

19. Lorigo LM, Faugeras OD, Grimson WEL, Keriven R, Kikinis R, Nabavi A, Westin CF (2001) CURVES: Curve evolution for vessel segmentation. Med. Image Anal. 5:195-200

20. Rennie MY, Detmar J, Whiteley KJ, Yang J, Jurisicova A, Adamson SL, Sled JG (2011) Vessel tortuousity and reduced vascularization in the fetoplacental arterial tree after maternal exposure to polycyclic aromatic hydrocarbons. Am. J. of Physiol. - Heart and Circulatory Physiol. 300:675-684

21. Cahill LS, Rennie MY, Hoggarth J, Yu LX, Rahman A, Kingdom JC, Seed M, Macgowan CK, Sled JG (2018) Feto- and utero-placental vascular adaptations to chronic maternal hypoxia in the mouse. The J. of Physiol. 596:3285-3297

22. Cheng Y, Hu X, Wang J, Wang Y, Tamura S (2015) Accurate vessel segmentation with constrained B-snake. Trans. Image Process. 24(8):2440-2455

23. Esneault S, Lafon C, Dillenseger JL (2010) Liver Vessels Segmentation Using a Hybrid Geometrical Moments/Graph Cuts Method. Trans. on BioMed. Eng. 57:276-283

24. Meijs M, Manniesing R (2018) Artery and vein segmentation of the cerebral vasculature in 4D CT using a 3D fully convolutional neural network. Med. Imaging 2018: Computer-Aided Diagn., SPIE

25. Zeng YZ, Zhao YQ, Liao M, Zou BJ, Wang XF, Wang W (2016) Liver vessel segmentation based on extreme learning machine. Phys. Medica 32:709-716

26. Anghel C, Archer K, Chang JM, Cochran A, Radulescu A, Salafia CM, Turner R, Djima KY, Zhong L (2018) Placental Vessel Extraction with Shearlets, Laplacian Eigenmaps, and a Conditional Generative Adversarial Network. Understanding Complex Biological Systems with Mathematics, Springer, Cham, 171-196pp

27. López-Linares K, Aranjuelo N, Kabongo L, Maclair G, Lete N, Ceresa M, García-Familiar A, Macía I, González Ballester MA (2018) Fully automatic detection and segmentation of abdominal aortic thrombus in post-operative CTA images using deep convolutional neural networks. Med. Img. Anal. 46: 202-214

28. Guo P, Wang Q, Wang X, Hao Z, Xu K, Ren H, Kim JB, Hwang Y (2014) Robust vessel detection and segmentation in ultrasound images by a data-driven approach. SPIE Med. Imaging 2014, 9034:903435

29. Schneider C, Guerrero J, Nguan C, Rohling R, Salcudean S (2011) Intra-operative "Pick-Up" Ultrasound for Robot Assisted Surgery with Vessel Extraction and Registration: A Feasibility Study. IPCAI 2011 122-132

30. Torrents-Barrena J, López-Velazco R, Piella G, Masoller N, Valenzuela-Alcaraz B, Gratacós E, Eixarch E, Ceresa M, González Ballester MA (2019) TTTS-GPS: Patient-specific preoperative planning and simulation platform for twin-to-twin transfusion syndrome fetal surgery. Comput. Methods and Programs in Biomed 179:104993

31. Torrents-Barrena J, Piella G, Masoller N, Gratacos E, Eixarch E, Ceresa M, Gonzalez Ballester MA (2019) Automatic Segmentation Of the Placenta and its Peripheral Vasculature in Volumetric Ultrasound for TTTS Fetal Surgery. 2019 IEEE 16th Int. Symposium on Biomed. Img. (ISBI 2019) 772-775

32. Grady L (2006) Random Walks for Image Segmentation. Trans. on Pattern Anal. and Mach. Intell. 28:17681783

33. Jianbing S, Yunfan D, Wenguan W, Xuelong L (2014) Lazy Random Walks for Superpixel Segmentation. Trans. on Med. Image Process. 23:1451-1462

34. Pujadas ER, Kjer HM, Piella G, González Ballester, MA (2016) Iterated random walks with shape prior. Image and Vis. Comput. 54:12-21

35. McCormick M, Liu X, Jomier J, Marion C, Ibanez L (2014) ITK: enabling reproducible research and open science. Front Neuroinform. 8:13

36. Guennebaud G, Jacob B, Bossart R, Gomez Ferrero D, Nuentsa D, and others (2010) Eigen3. http://eigen.tuxfamily.org

37. van der Vorst HA (1992) Bi-CGSTAB: A Fast and Smoothly Converging Variant of Bi-CG for the Solution of Nonsymmetric Linear Systems. SIAM J. on Scientific and Statistical Comput. 13(2):631-644 
38. Gocławski J, Weffgliński T, Fabijańska A (2015) Accelerating the 3D Random Walker Image Segmentation Algorithm by Image Graph Reduction and GPU Computing. In: Image Processing Communications Challenges 6, Springer, Cham, pp 45-52 